\title{
Enhancing DNA vaccine potency by coadministration of DNA encoding antiapoptotic proteins
}

\author{
Tae Woo Kim, ${ }^{1}$ Chien-Fu Hung, ${ }^{1}$ Morris Ling, ${ }^{1}$ Jeremy Juang, ${ }^{1}$ Liangmei He, ${ }^{1}$ \\ J. Marie Hardwick, ${ }^{2,3,4,5}$ Sharad Kumar, ${ }^{6}$ and T.-C. Wu ${ }^{1,2,5,7}$ \\ ${ }^{1}$ Department of Pathology, \\ ${ }^{2}$ Department of Molecular Microbiology and Immunology, \\ ${ }^{3}$ Department of Pharmacology and Molecular Sciences, \\ ${ }^{4}$ Department of Neurology, \\ ${ }^{5}$ Department of Oncology, The Johns Hopkins Medical Institutions, Baltimore, Maryland, USA \\ ${ }^{6} \mathrm{~J}$. Hanson Centre for Cancer Research, Institute of Medical and Veterinary Science, Adelaide, Australia \\ ${ }^{7}$ Department of Obstetrics and Gynecology, the Johns Hopkins Medical Institutions, Baltimore, Maryland, USA
}

\begin{abstract}
Intradermal vaccination by gene gun efficiently delivers DNA vaccines into DCs of the skin, resulting in the activation and priming of antigen-specific T cells in vivo. DCs, however, have a limited life span, hindering their long-term ability to prime antigen-specific $\mathrm{T}$ cells. We reason that a strategy that prolongs the survival of DNA-transduced DCs will enhance priming of antigen-specific $T$ cells and DNA vaccine potency. Here we show that codelivery of DNA encoding inhibitors of apoptosis (BCL-xL, BCL-2, XIAP, dominant negative caspase-9, or dominant negative caspase-8) with DNA encoding model antigens prolongs the survival of transduced DCs. More importantly, vaccinated mice exhibited significant enhancement in antigen-specific $\mathrm{CD}^{+} \mathrm{T}$ cell immune responses, resulting in a potent antitumor effect against antigen-expressing tumors. Among these antiapoptotic factors, $\mathrm{BCL}-\mathrm{xL}$ demonstrated the greatest enhancement in antigen-specific immune responses and antitumor effects. Thus, coadministration of DNA vaccines with DNA encoding antiapoptotic proteins represents an innovative approach to enhance DNA vaccine potency.
\end{abstract}

J. Clin. Invest. 112:109-117 (2003). doi:10.1172/JCI200317293.

\section{Introduction}

Intradermal administration of DNA vaccines using a gene gun can be used to efficiently deliver genes of interest into professional APCs in vivo (1). The skin contains numerous bone marrow-derived APCs (called Langerhans cells) that are able to move through the lymphatic system from the site of injection to draining lymph nodes, where they prime antigen-specific T cells (2). Gene gun immunization therefore provides the opportunity to test vaccine strategies that require direct delivery of DNA to APCs. Previously, we used the gene gun approach to test several intracellular targeting strategies that are able to route model

Received for publication November 1, 2002, and accepted in revised form March 11, 2003

Address correspondence to: T.-C. Wu, Department of Pathology, The Johns Hopkins University School of Medicine, Ross $512 \mathrm{H}$, 720 Rutland Avenue, Baltimore, Maryland 21205, USA. Phone: (410) 614-3899; Fax: (443) 287-4295;

E-mail: wutc@jhmi.edu.

Tae Woo Kim and Chien-Fu Hung contributed equally to this work.

Conflict of interest: The authors have declared that no conflict of interest exists.

Nonstandard abbreviations used: human Papillomavirus type-16 (HPV-16); X-linked inhibitor of apoptosis protein (XIAP); dominant negative (dn); phycoerythrin (PE); mutant BCL-xL (mt 7); hemagglutinin (HA); cytosine linked to a guanine by a phosphate bond $(\mathrm{CPG})$. antigen (human Papillomavirus type-16 [HPV-16] E7) to desired subcellular compartments and enhance antigen processing and presentation to $T$ cells (3-6). We also used this system to develop strategies that lead to intercellular spreading of antigen from initially transfected cells to surrounding APCs, resulting in enhanced E7-specific $\mathrm{CD}^{+} \mathrm{T}$ cell-mediated immune responses $(7,8)$. Therefore, direct delivery of DNA vaccines into DCs by a gene gun provides an opportunity to modify the quality and quantity of DNA-transfected DCs and influence vaccine potency.

One potential strategy to further enhance DNA vaccine potency is to prolong DC survival by coadministering DNA encoding antigen with DNA encoding inhibitors of apoptosis to DCs. In this study, we tested a variety of antiapoptotic factors for their ability to enhance DC survival and E7-specific $\mathrm{CD}^{+} \mathrm{T}$ cell immune responses when coadministered with E7 DNA, including BCL-xL $(9,10)$ and BCL-2 $(11,12)$, members of the BCL-2 family of proteins; X-linked inhibitor of apoptosis protein (XIAP) (13); and dominant negative (dn) mutants of caspases such as dn caspase-9 (14) and dn caspase-8 (15), which have a mutation in the enzyme active site and serve as inhibitors of apoptosis. We also evaluated the effect of mutant BCL-xL ( $\mathrm{mt} 7$ ) (10), which has mutations in a region critical to antiapoptotic function (aa 135-137 in the BH1 domain), and caspase-3 (16), a proapoptotic pro- 
tein when it is coadministered with DNA encoding antigen. We chose HPV-16 E7 as a model antigen because HPVs, particularly HPV-16, are associated with a majority of cervical cancers, and E7 is essential for the induction and maintenance of cellular transformation. Effective vaccines against E7 potentially can be used for the control of HPV infections and HPV-associated lesions. By examining E7-specific immune responses, antitumor effects, survival of DNA-transfected DCs, and the ability of DNA-transfected DCs to activate an E7-specific $T$ cell line, we demonstrate that coadministration of antigen-containing DNA with DNA encoding antiapoptotic proteins represents an innovative strategy to enhance vaccine potency.

\section{Methods}

Plasmid DNA constructs and DNA preparation. The generation of pcDNA3-E7 (4), pCMV(neo)-Sig/E7/LAMP-1 (3), and pDNA3-E7/GFP (5) has been described previously. The plasmid containing influenza hemagglutinin (HA), pcDNA3-HA, was kindly provided by Drew Pardoll at the Johns Hopkins School of Medicine. The pEBB-XIAP (17), pcDNA3-FLICEc-s (15), and pSG5 plasmids encoding BCL-xL (10), mt 7 (our mutant BLC-xL) (10), BCL-2 (18), or dn caspase-9 (19) have been generated in J. Marie Hardwick's lab. To generate pcDNA3-Sig/E7/LAMP-1, Sig/E7/LAMP-1 was isolated from pCMV(neo)-Sig/E7/LAMP-1 (3) and cloned into the EcoRI/BamHI sites of pcDNA3. For the generation of pcDNA3-OVA, the DNA fragment encoding OVA was amplified by a set of primers, $5^{\prime}$-CCCGAATTCATGGGCTCCATCGGCGCAGC- $3^{\prime}$ and $5^{\prime}$-CCCGGATCCAAATTCTTCAGAGACGCTTGC- $3^{\prime}$, and OVA cDNA, a generous gift from Michael Bevan at the University of Washington School of Medicine (Seattle, Washington, USA). The amplified product was further cloned into the EcoRI/BamHI sites of pcDNA3. For the generation of pSG5-XIAP, the DNA fragment encoding XIAP was amplified with PCR using PEBB-XIAP as template and a set of primers: 5'-GCTAGGATCCATGACTTTTAACAGTTTTGAAGG- $3^{\prime}$ and $5^{\prime}$-GCACGGATCCTTAAGACATAAAAATTTTTTGCT- $3^{\prime}$. The amplified product was further cloned into the BamHI cloning site of pSG5. For the generation of PSG5-dn caspase-8, the DNA fragment of dn caspase-8 was amplified with PCR using pcDNA3-FLICEc-s as a template and a set of primers, 5'-GCTAGGATCCATGGACTTCAGCAGAAATCTTT- ${ }^{\prime}$ and $5^{\prime}$-GCACGGATCCTCAATCAGAAGGGAAGACAAG- $3^{\prime}$. The amplified product was further cloned into the BamHI cloning site of PSG5. For the generation of PSG5-caspase- 3 and pSG5-mt caspase-3, the DNA fragments of caspase- 3 and its mutant were amplified with PCR using C2P-caspase-3-GFP and C2P-caspase$3 \Delta 9$ (C163)-GFP (20) as a template, respectively, and a set of primers, $5^{\prime}$-CCGTCAGATCCGCTAGCGCTACCGG$3^{\prime}$ and $5^{\prime}$-GTGCATCCCTTAGGTGATAAAAATAGAGTTC$3^{\prime}$. The amplified product was further cloned into the BamHI sites of PSG5. The accuracy of these constructs was confirmed by DNA sequencing. The DNA was amplified in Escherichia coli DH5 $\alpha$ and purified as described previously (4).

Western blot analysis. The expression of proapoptotic and antiapoptotic proteins in COS-7 cells transfected with DNA encoding antiapoptotic protein was characterized by Western blot analysis. The DNA encoding the various proapoptotic and antiapoptotic proteins also contains an HA epitope (YPYDBPDYA) at the $5^{\prime}$ end of the encoded protein to serve as a tag. Western blot analysis was performed with $50 \mu \mathrm{g}$ of the cell lysate derived from COS-7 cells transfected with the various DNA constructs encoding the proapoptotic and antiapoptotic proteins and anti-HA mouse $\mathrm{mAb}$ (clone12CA5; Roche Diagnostics Corp., Indianapolis, Indiana, USA) using the method described previously (5).

Mice. Six- to eight-week-old female C57BL/6 mice were purchased from the National Cancer Institute (Frederick, Maryland, USA) and kept in the oncology animal facility of the Johns Hopkins Hospital (Baltimore, Maryland, USA). All animal procedures were performed according to approved protocols and in accordance with recommendations for the proper use and care of laboratory animals.

DNA vaccination. DNA-coated gold particles were prepared according to a protocol described previously (4). DNA-coated gold particles were delivered to the shaved abdominal region of mice using a helium-driven gene gun (Bio-Rad Laboratories Inc., Hercules, California, USA) with a discharge pressure of 400 psi. C57BL/ 6 mice were immunized with $2 \mu \mathrm{g}$ of the plasmid encoding E7, Sig/E7/LAMP-1, HA, or OVA mixed with $2 \mu \mathrm{g}$ of pSG5, pSG5-BCL-xL, pSG5-XIAP, pSG5-BCL-2, pSG5-dn caspse-9, pSG5-dn caspase-8, pSG5-mt BCL-xL, pSG5-caspase-3, or pSG5-mt caspase-3. The mice received a booster with the same dose 1 week later.

Intracellular cytokine staining and flow-cytometry analysis. Splenocytes were harvested from mice 1 week after the last vaccination. Prior to intracellular cytokine staining, $4 \times 10^{6}$ pooled splenocytes from each vaccination group were incubated for 16 hours with either $1 \mu \mathrm{g} / \mathrm{ml}$ of E7 (RAHYNIVTF), HA (IYSTVASSL), or OVA peptide (SIINFEKL) containing an MHC class I epitope for detecting antigen-specific $\mathrm{CD}^{+} \mathrm{T}$ cell precursors. Intracellular IFN- $\gamma$ staining and flow-cytometry analysis were performed as described previously (4). Analysis was performed on a Becton-Dickinson FACScan with CELLQuest software (Becton Dickinson Immunocytometry Systems, Mountain View, California, USA).

In vivo tumor protection and tumor-treatment experiments. The HPV-16 E7-expressing murine tumor model, TC-1, has been described previously (21). In brief, HPV-16 E6, $\mathrm{E} 7$, and ras oncogene were used to transform primary C57BL/6 mice-lung epithelial cells to generate TC-1. For the tumor-protection experiment, C57BL/ 6 mice (five per group) were subcutaneously challenged with $5 \times 10^{4} \mathrm{TC}-1$ tumor cells per mouse in the right leg 1 week after the last vaccination. Mice were monitored for evidence of tumor growth by palpation and inspection twice a week. To study the subset of lymphocytes 
that are important for the antitumor effects, an in vivo $\mathrm{Ab}$ depletion experiment was performed using the method described previously (21).

For the tumor-treatment experiment, mice were challenged with $10^{4} \mathrm{TC}-1$ tumor cells per mouse in the tail vein to simulate hematogenous spread of tumors (3). Mice were treated with DNA 3 days after tumor challenge. Mice were monitored twice a week and sacrificed on day 42 after the last vaccination. The mean number of pulmonary nodules in each mouse was evaluated by experimenters blinded to sample identity. In vivo tumor protection, $\mathrm{Ab}$ depletion, and tumor-treatment experiments were performed three times to generate reproducible data.

Preparation of $\mathrm{CD} 11 c^{+}$cells in the inguinal lymph nodes from vaccinated mice. C57BL/6 mice (three per group) received 12 inoculations of nonoverlapping intradermal administration with a gene gun on the abdominal region. Gold particles used for each inoculation were coated with $1 \mu \mathrm{g}$ of pcDNA3-E7/GFP DNA mixed with $1 \mu \mathrm{g}$ of pSG5 encoding BCL-xL, mt BCL-xL, caspase-3, or no insert. The pcDNA3 (no insert) mixed with pSG5-BCL-xL was used as a negative control. Inguinal lymph nodes were harvested from vaccinated mice 1 or 5 days after vaccination with a gene gun. A single cell suspension from isolated inguinal lymph nodes was prepared. CD11 $\mathrm{c}^{+}$cells were enriched from lymph nodes using CD11c (N418) microbeads (Miltenyi Biotec, Auburn, California, USA). Enriched CD11 $\mathrm{c}^{+}$ cells were analyzed by forward and side scatter and gated around a population of cells with size and granular characteristics of DCs. The percentage of CD11 $\mathrm{c}^{+}$ cells in the gated area was characterized by flow-cytometry analysis using phycoerythrin-conjugated (PE-conjugated) anti-CD11c Ab (PharMingen, San Diego, California, USA). GFP-positive cells were analyzed by flow-cytometry analysis using a protocol described previously (22). Data are expressed as percentage of

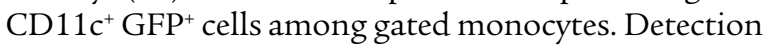
of apoptotic cells in the $\mathrm{CD} 11 \mathrm{c}^{+} \mathrm{GFP}^{+}$cells was performed using annexin V-PE apoptosis detection Kit-I (BD Bioscience, San Diego, California, USA) according to the vendor's protocol. The percentage of apoptotic cells was analyzed using flow-cytometry analysis by gating $\mathrm{CD} 11 \mathrm{c}^{+} \mathrm{GFP}^{+}$cells.

Activation of an E7-specific CD8 $\mathrm{T}$ cell line by $\mathrm{CD} 11 c^{-}$ enriched cells from vaccinated mice. Mice were vaccinated, and enriched CD11 $\mathrm{c}^{+}$cells were collected as described above. CD11c-enriched cells $\left(2 \times 10^{4}\right)$ were incubated with $2 \times 10^{6}$ of the E7-specific $\mathrm{CD}^{+} \mathrm{T}$ cell line (23) for 16 hours. Cells were then stained for both surface CD8 and intracellular IFN- $\gamma$ and analyzed with flow-cytometry analysis as described above.

\section{Results}

Coadministration of E7 DNA with DNA encoding antiapoptotic factors significantly enhances E7-specific $C D 8^{+}$T cell-mediated immune responses. We hypothesized that DNA encoding antiapoptotic proteins would enhance $\mathrm{E} 7$-specific $\mathrm{CD}^{+}$
T cell immune responses when coadministered with E7 DNA. We have therefore generated DNA constructs encoding antiapoptotic proteins. Expression of antiapoptotic proteins was confirmed in transfected COS-7 cells by Western blot analysis (data not shown). The expression levels of wild-type and mutant forms of the proteins are equivalent (data not shown).

To assess the quantity of E7-specific $\mathrm{CD}^{+} \mathrm{T}$ cell precursors generated by vaccination with E7 DNA mixed with DNA encoding antiapoptotic or proapoptotic proteins, we performed intracellular cytokine staining with flow-cytometry analysis. As shown in Figure 1, a and b, mice vaccinated with E7 DNA mixed with BCL-xL DNA generated the highest frequency of E7-specific IFN- $\gamma$ secreting $\mathrm{CD}^{+} \mathrm{T}$ cell precursors $\left(58.3 \pm 9.5 / 3 \times 10^{5}\right.$ splenocytes), more than 11-fold greater than the number of precursors in mice vaccinated with E7 DNA mixed with pSG5 (no insert) $\left(5.0 \pm 1.0 / 3 \times 10^{5}\right.$ splenocytes $)(P<0.01)$. Similarly, vaccination with E7 DNA mixed with DNA encoding other antiapoptotic proteins also led to an increase in the number of E7-specific $\mathrm{CD}^{+} \mathrm{T}$ cells: E7 plus XIAP $\left(50.7 \pm 3.8 / 3 \times 10^{5}\right.$ splenocytes); E7 plus BCL-2 (48.7 $\pm 3.1 / 3 \times 10^{5}$ splenocytes); E7 plus dn caspase- $9\left(28.0 \pm 3.0 / 3 \times 10^{5}\right.$ splenocytes); and E7 plus dn caspase-8 $\left(23.7 \pm 1.5 / 3 \times 10^{5}\right.$ splenocytes). In contrast, coadministration of E7 DNA with DNA encoding a proapoptotic protein, caspase-3, did not augment the number of E7-specific CD8 ${ }^{+} \mathrm{T}$ cell precursors $\left(2.3 \pm 0.6 / 3 \times 10^{5}\right.$ splenocytes). Our results also indicated that $\mathrm{E} 7$ antigen was required for this immune-enhancing effect since pcDNA3 (no insert) coadministered with BCL-xL did not enhance E7-specific $\mathrm{CD}^{+} \mathrm{T}$ cell activity $\left(4.3 \pm 2.1 / 3 \times 10^{5}\right.$ splenocytes). These data indicate that coadministration of E7 DNA with DNA encoding antiapoptotic factors can lead to a marked increase in the number of E7-specific $\mathrm{CD}^{+} \mathrm{T}$ cell precursors.

Vaccination with E7 DNA mixed with DNA encoding antiapoptotic protein leads to E7-specific tumor protection. To determine if the observed enhancement in E7-specific $\mathrm{CD}^{+} \mathrm{T}$ cell-mediated immunity leads to a significant E7-specific antitumor effect, we performed an in vivo tumor-protection experiment using a previously described E7-expressing tumor model, TC-1 (21). As shown in Figure 1c, $80 \%$ of mice receiving E7 DNA mixed with BCL-xL DNA remained tumor free 46 days after TC-1 challenge. In contrast, all of the mice receiving E7 DNA mixed with PSG5 (no insert) or caspase-3 developed tumors by day 46 . Similarly, we observed that coadministration of DNA encoding either XIAP or BCL-2, like BCL-xL, can also result in significant antitumor effects by inhibiting tumor formation in the subcutaneous tumor model (data not shown).

We have also performed an in vivo Ab depletion experiment to determine the subsets of lymphocytes important for the antitumor effects. As shown in Figure $1 \mathrm{~d}, 100 \%$ of the mice depleted of $\mathrm{CD}^{+} \mathrm{T}$ cells grow tumors within 2 weeks after TC- 1 challenge. In contrast, $100 \%$ of the mice depleted of $\mathrm{CD}^{+} \mathrm{T}$ cells or $\mathrm{NK}$ 
cells remained tumor free 42 days after TC- 1 challenge. These data indicated that $\mathrm{CD}^{+} \mathrm{T}$ cells are important for the antitumor effect generated by the DNA vaccine. We also performed an in vivo tumor-treatment experiment using a hematogenous spread pulmonary tumor model (3). As shown in Figure 1e, mice immunized with E7 DNA mixed with BCL-xL exhibited the fewest number of pulmonary tumor nodules $(1.2 \pm 1.3$; $P<0.001$, one-way ANOVA) compared with mice vaccinated with E7 DNA mixed with caspase-3 $(11.6 \pm 4.7)$, E7 DNA mixed with pSG5 (no insert) $(8.0 \pm 2.5)$, or pcDNA3 (no insert) mixed with BCL-xL $(11.8 \pm 2.4)$. We also observed that coadministration of DNA encoding either XIAP or BCL-2, like BCL-xL, can result in significant antitumor effects by inhibiting tumor nodule formation in the hematogenous spread pulmonary tumor model (data not shown). Taken together, these results indicate that vaccination with E7 DNA mixed with DNA encoding antiapoptotic proteins leads to potent protective and therapeutic effects against E7-expressing TC-1 tumor cells.

Coadministration of DNA encoding HA or OVA with DNA encoding antiapoptotic protein leads to enhanced antigen-specific $C D 8^{+} T$ cell immune responses. To determine if the observed enhancement of $\mathrm{CD}^{+} \mathrm{T}$ cell-mediated immunity is a general phenomenon that is applicable to other antigens, we used different antigen-expressing DNA vaccines in combination with DNA encoding antiapoptotic protein. Mice were immunized with pcDNA3 containing well-characterized antigens, $\mathrm{HA}$ or OVA, mixed with pSG5 DNA containing no insert or BCL-xL. Using intracellular cytokine staining with flowcytometry analysis, we found that vaccination with pcDNA3-HA or pcDNA3-OVA mixed with BCL-xL

\section{Figure 1}

E7-specific CD8 ${ }^{+} \mathrm{T}$ cell immune responses and antitumor effect generated by vaccination with E7 DNA mixed with DNA encoding antiapoptotic or proapoptotic proteins. The pcDNA3 (no insert) mixed with pSG5-BCL-xL was used as a negative control. (a) Representative figure of the flow-cytometry data. The data presented in this figure are from one representative experiment of three performed. (b) Bar graph depicting the number of antigen-specific IFN- $\gamma$-secreting $\mathrm{CD}^{+}$ T cell precursors per $3 \times 10^{5}$ splenocytes (mean $\pm \mathrm{SD}$ ). (c) In vivo tumor-prevention experiment. Mice were immunized with pcDNA3-E7 mixed with pSG5 encoding BCL-xL, caspase-3, or no insert. The PcDNA3 (no insert) mixed with pSG5-BCL-xL was used as a negative control. One week after the last vaccination, mice were subcutaneously challenged with $5 \times 10^{4}$ TC- 1 cells per mouse in the right leg. (d) In vivo Ab-depletion experiments to determine the contribution of various lymphocyte subsets on the tumor protection generated by the coadministration of pcDNA3-E7 and PSG5-BCL-xL DNA vaccine. CD4, CD8, and NK1.1 depletions were initiated 1 week before tumor challenge. (e) In vivo tumor-treatment experiment. Mice received $10^{4} \mathrm{TC}-1$ tumor challenge and were immunized 3 days later with pcDNA3-E7 mixed with pSG5 encoding $B C L-x L$, caspase-3, or no insert. In vivo tumor protection, $\mathrm{Ab}$ depletion, and tumor-treatment experiments were performed three times. Casp, caspace.
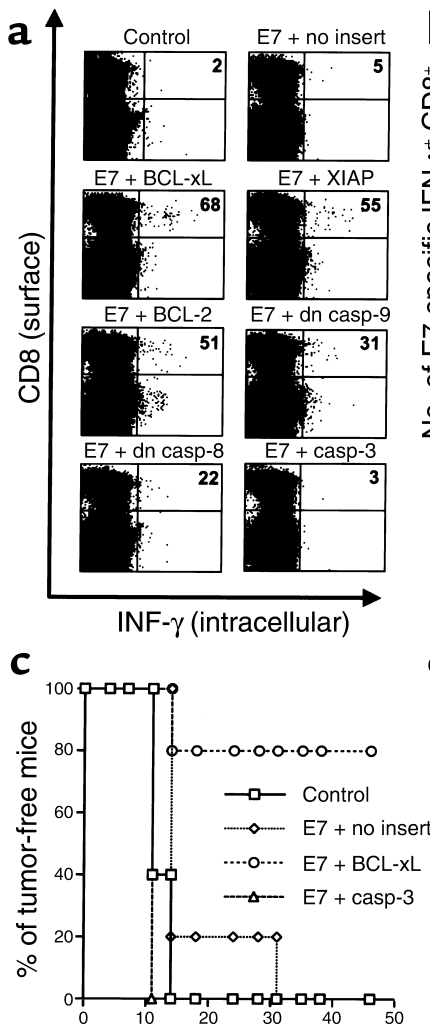

Days after TC- 1 challenge

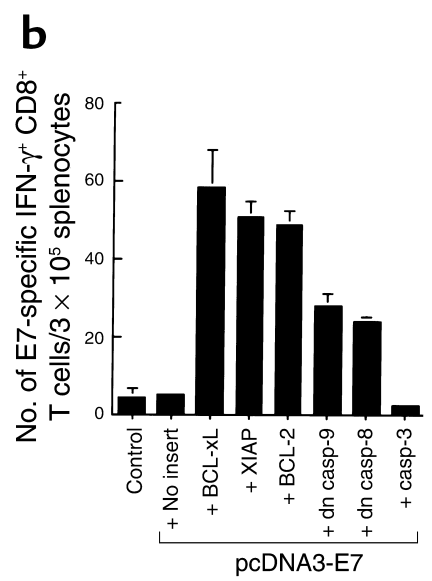

d
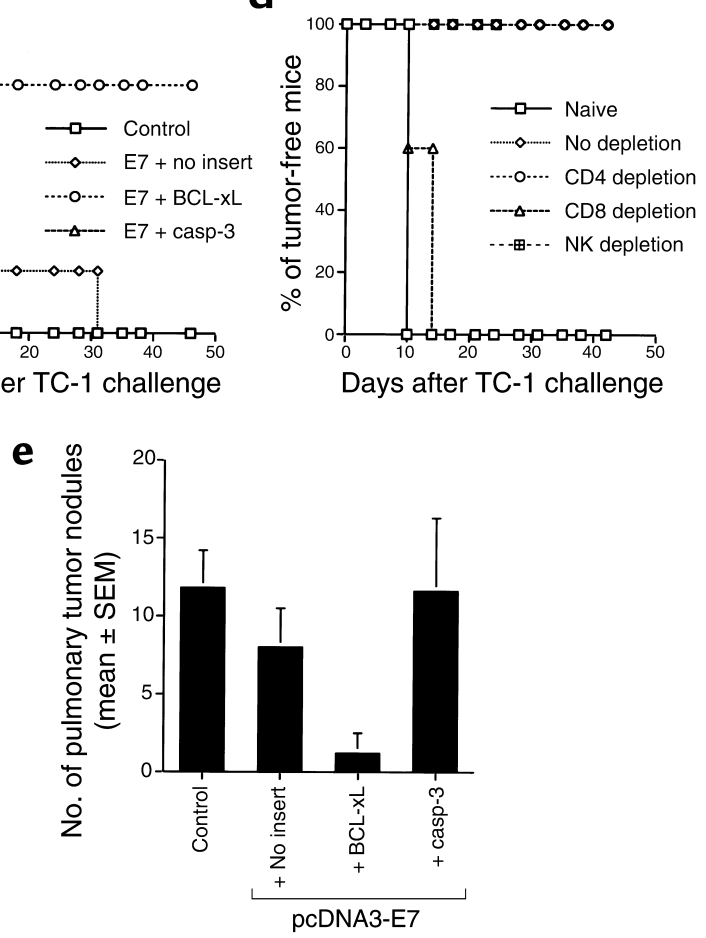


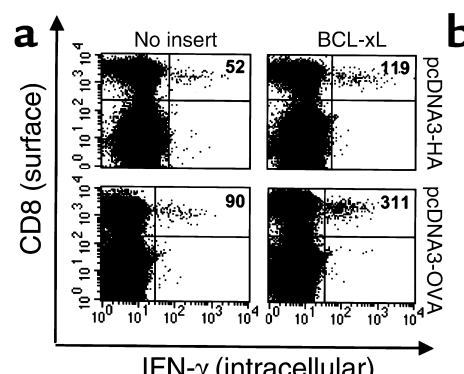

IFN- $\gamma$ (intracellular)
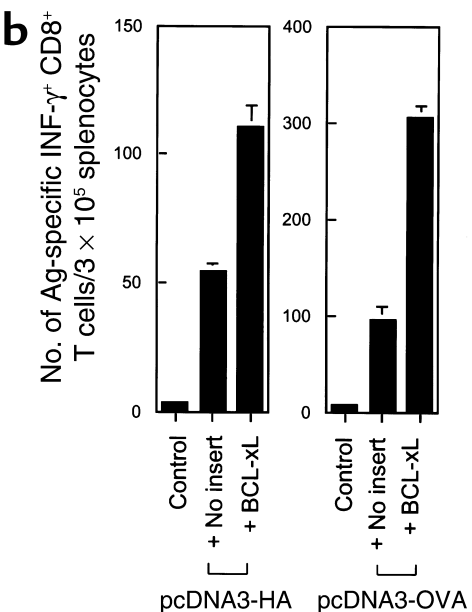

\section{Figure 2}

Antigen-specific $\mathrm{CD}^{+} \mathrm{T}$ cell precursors in mice vaccinated with DNA encoding HA or OVA coadministered with DNA encoding an antiapoptotic protein. Mice (three per group) were immunized with pcDNA3 containing HA or OVA mixed with pSG5 encoding an antiapoptotic gene (BCL-xL) or no insert. The pcDNA3 (no insert) mixed with pSG5-BCL-xL was used as a negative control. Splenocytes were collected and prepared as described in the legend of Figure 1. (a) Representative set of the flow-cytometry data. The data presented in this figure are from one representative experiment of three performed. (b) Bar graph depicting the number of antigenspecific IFN- $\gamma$-secreting $C D 8^{+} T$ cell precursors per $3 \times 10^{5}$ splenocytes (mean $\pm \mathrm{SD}$ ).
DNA significantly increased the number of antigenspecific $\mathrm{CD}^{+} \mathrm{T}$ cell precursors compared with vaccination of pcDNA3-HA or pcDNA3-OVA mixed with pSG5 (no insert), respectively (Figure 2, a and b). These results suggest that coadministration of DNA encoding different model antigens with DNA encoding an antiapoptotic protein can lead to an increase in the number of antigen-specific $\mathrm{CD}^{+} \mathrm{T}$ cell precursors.

The combined application of antiapoptosis and an intracellular targeting strategy leads to enhancement of E7-specific $\mathrm{CD} 8^{+} \mathrm{T}$ cell immune responses. We have developed vaccines that target antigen intracellularly to desired subcellular compartments and enhance MHC class I and/or class II presentation of antigen to $\mathrm{CD}^{+}$and $\mathrm{CD}^{+}$ $\mathrm{T}$ cells, respectively $(3,4,6)$. One such vaccine, Sig/E7/LAMP-1 DNA (signal peptide/E7/lysosomeassociated membrane protein) is able to target E7 to the endosomal/lysosomal compartments, which have been shown to enhance MHC class II presentation of E7 to $\mathrm{CD}^{+} \mathrm{T}$ cells and also increase the number of E7specific $\mathrm{CD} 8^{+} \mathrm{T}$ cells to prevent the development of E7expressing tumors in mice (3). To assess the effect of DNA encoding antiapoptotic proteins when coadministered with DNA encoding E7 linked to an immunogenic molecule, mice were vaccinated with Sig/E7/ LAMP-1 DNA mixed with DNA encoding different antiapoptotic or proapoptotic proteins. As shown in Figure 3, a and b, vaccination with Sig/E7/LAMP-1 DNA coadministered with BCL-xL DNA generated the highest frequency of E7-specific IFN- $\gamma$-secreting $\mathrm{CD}^{+}$ $\mathrm{T}$ cell precursors $\left(1,752.7 \pm 99.9 / 3 \times 10^{5}\right.$ splenocytes $)$, greater than the number of precursors observed in mice vaccinated with Sig/E7/LAMP-1 DNA mixed with pSG5 (no insert) $\left(167.3 \pm 16.2 / 3 \times 10^{5}\right.$ splenocytes) $(P<0.01$, ANOVA) or E7 DNA mixed with pSG5-BCL$\mathrm{xL}\left(58.3 \pm 9.5 / 3 \times 10^{5}\right.$ splenocytes) (see Figure 1$)$. Similarly, combined vaccination of Sig/E7/LAMP-1 DNA with other DNA encoding other antiapoptotic proteins increased $\mathrm{E} 7$-specific $\mathrm{CD} 8^{+} \mathrm{T}$ cell precursor numbers: Sig/E7/LAMP-1 plus XIAP $(1,530.7 \pm 115.6 /$ $3 \times 10^{5}$ splenocytes), Sig/E7/LAMP-1 plus BCL-2
(1,462.7 $\pm 99.9 / 3 \times 10^{5}$ splenocytes), Sig/E7/LAMP-1 plus dn caspase-9 $\left(619.7 \pm 62.1 / 3 \times 10^{5}\right.$ splenocytes $)$, and Sig/E7/LAMP-1 plus dn caspase-8 (430.0 \pm 25.9 / $3 \times 10^{5}$ splenocytes).

We have also determined whether the class II presentation of $\mathrm{CD}^{+} \mathrm{T}$ cells is enhanced in the presence of BCL-xL. Our results indicated that mice vaccinated with pcDNA3-Sig/E7/LAMP-1 mixed with pSG5-BCL-xL demonstrated significantly higher numbers of E7-specific $\mathrm{CD}^{+} \mathrm{T}$ cells (sixfold higher) than mice vaccinated with pcDNA3-Sig/E7/LAMP-1 mixed with pSG5 (data not shown).

Coadministration of Sig/E7/LAMP-1 DNA with DNA encoding mutant $B C L-x L$, caspase-3, or $m t$ caspase- 3 fails to lead to efficient activation of E7-specific $C D 8^{+}$Tcell activity by gene gun administration. We evaluated the effect of a mutation abrogating the antiapoptotic function of BCL-xL. While we found that vaccination with Sig/E7/LAMP-1 DNA mixed with BCL-xL DNA led to a marked increase in the number of E7-specific IFN- $\gamma$ secreting $\mathrm{CD}^{+} \mathrm{T}$ cell precursors $\left(1,816 \pm 54.7 / 3 \times 10^{5}\right.$ splenocytes), we did not observe the same phenomenon with DNA encoding defective mutant BCL-xL (pSG5mt BCL-xL) $\left(168 \pm 16.3 / 3 \times 10^{5}\right.$ splenocytes; $P<0.001$, ANOVA) (Figure 3, c and d). In addition, we showed that the coadministration of pcDNA3-Sig/E7/LAMP-1 with DNA encoding a proapoptotic protein, wild-type, or mutated caspase-3 (which has been shown to demonstrate less proapoptotic function; ref. 24) led to a significant decrease in E7-specific $\mathrm{CD}^{+} \mathrm{T}$ cell precursor numbers $\left(5 \pm 1.3 / 3 \times 10^{5}\right.$ and $52 / 9.7 \times 10^{5}$ splenocytes, respectively) compared with mice vaccinated with Sig/E7/LAMP-1 DNA mixed with DNA encoding PSG5 (no insert). Our results suggest that the antiapoptotic function of BCL-xL is critical for the observed enhancement of the E7-specific $\mathrm{CD}^{+} \mathrm{T}$ cell immune response mediated by the coadministration of E7 DNA with DNA encoding BCL-xL.

Coadministration of Sig/E7/LAMP-1 DNA with DNA encoding BCL-xL generates long-term E7-specific $C D 8^{+} T$ cell memo$r y$. We further evaluated the E7-specific $\mathrm{CD}^{+} \mathrm{T}$ cell 
immune response in mice vaccinated with various combinations of DNA constructs at 1, 7,12, and 14 weeks after the last DNA vaccination. As shown in Figure 3e, mice vaccinated with pcDNA3-Sig/E7/LAMP-1 mixed with PSG5-BCL-xL generated consistently the highest numbers of E7-specific $\mathrm{CD}^{+} \mathrm{T}$ cell precursors throughout the duration of the experiment compared with mice vaccinated with pcDNA3-Sig/E7/LAMP-1 DNA mixed with pSG5 DNA or PSG5-caspase-3. Thus, our data suggest that the coadministration of Sig/E7/LAMP-1 DNA with DNA encoding BCL-xL is capable of generating long-term E7-specific CD8 ${ }^{+} \mathrm{T}$ cell memory.

Improved survival of DNA-transfected DCs in inguinal lymph nodes of mice vaccinated with E7/GFP DNA mixed with DNA encoding antiapoptotic protein. Following intradermal immunization, DCs are known to migrate to draining lymph nodes where they stimulate antigenspecific T cells $(1,2)$. We used GFP linked to E7 to serve as a fluorescent tag to identify DNA-transfected DCs in the inguinal lymph nodes. Inguinal lymph nodes were harvested from vaccinated mice 1 and 5 days after gene gun vaccination. Because $\mathrm{CD} 11 \mathrm{c}^{+}$cells also include myeloid cells other than DCs, such as NK cells and $\mathrm{B}$ and $\mathrm{T}$ cell subsets, we gated a region more consistent with DC size and granular characteristics as reported previously (22) in order to increase the percentage of $\mathrm{GFP}^{+} \mathrm{CD} 11 \mathrm{c}^{+} \mathrm{DC}$ for comparison between different vaccinated groups. We also stained for additional markers for DCs and found that more than $90 \%$ of the $\mathrm{GFP}^{+} \mathrm{CD} 11 \mathrm{c}^{+}$cells express DC surface markers such as B7.1 and B7.2 and CD40 (data not shown). As shown in Figure 4, a and b, there is no significant dif-

\section{Figure 3}

E7-specific CD8 ${ }^{+} \mathrm{T}$ cell immune responses in mice vaccinated with Sig/E7/LAMP-1 DNA mixed with DNA encoding antiapoptotic or proapoptotic proteins. Mice (three per group) were immunized with pcDNA3-Sig/E7/LAMP-1 mixed with PSG5 encoding antiapoptotic protein (BCL-xL, XIAP, BCL-2, dn caspase-9, dn caspase-8), proapoptotic protein (caspase-3), or no insert. The pcDNA3 (no insert) mixed with PSG5-BCL-xL was used as a negative control. Splenocytes were collected and prepared as described in the legend of Figure 1. The number of E7-specific IFN- $\gamma$-secreting CD8 $8^{+} \mathrm{T}$ cell precursors was analyzed by intracellular cytokine staining followed by flowcytometry analysis. (a) Representative set of the flow-cytometry data. The data presented in this figure are from one representative experiment of three performed. (b) The bar graph depicts the number of E7-specific IFN- $\gamma$-secreting CD $8^{+} T$ cell precursors per $3 \times 10^{5}$ splenocytes (mean \pm SD). (c) Mice (three per group) were immunized with pcDNA3-Sig/E7/LAMP-1 mixed with pSG5 encoding BCL-xL, caspase-3, mt BCL-xL, mt caspase-3, or no insert. The pcDNA3 (no insert) mixed with $\mathrm{PSG} 5-\mathrm{BCL}-\mathrm{xL}$ was used as a negative control. The flow-cytometry data shown here are from one representative experiment of three performed. (d) Bar graph depicting the number of antigen-specific IFN- $\gamma$-secreting $C D 8^{+} T$ cell precursors per $3 \times 10^{5}$ splenocytes (mean $\pm \mathrm{SD}$ ). (e) Graph depicting the number of antigen-specific IFN- $\gamma$-secreting $C D 8^{+} T$ cell precursors was evaluated at $1,7,12$, and 14 weeks after coadministration of pcDNA-Sig/E7/LAMP-1 with pSG5$\mathrm{BCL}-\mathrm{xL}$, pSG5-caspase-3, or pSG5 (no insert). casp, caspase. ference in numbers of CD11c and GFP-positive cells in the inguinal lymph nodes at day 1 after vaccination with E7 DNA mixed with BCL-xL DNA or control plasmid. At day 5 after gene gun vaccination, however, we observed a greater percentage of $\mathrm{GFP}^{+} \mathrm{CD} 11 \mathrm{c}^{+}$cells in lymph nodes harvested from mice vaccinated with the E7/GFP DNA mixed with BCL-xL DNA than in lymph nodes harvested from mice vaccinated with E7/GFP DNA mixed with DNA encoding proapoptotic caspase-3, mt BCL-xL, or no insert $(P<0.0005$, oneway ANOVA) (Figure $4 b$ ). Furthermore, we assayed for the apoptotic cells in $\mathrm{CD} 11 \mathrm{c}^{+} \mathrm{GFP}^{+}$cells derived from the draining lymph nodes of various vaccinated mice by staining for annexin $V$ followed by flow-cytometry analysis. As shown in Figure 4c, mice vaccinated with DNA encoding E7/GFP mixed with DNA encoding
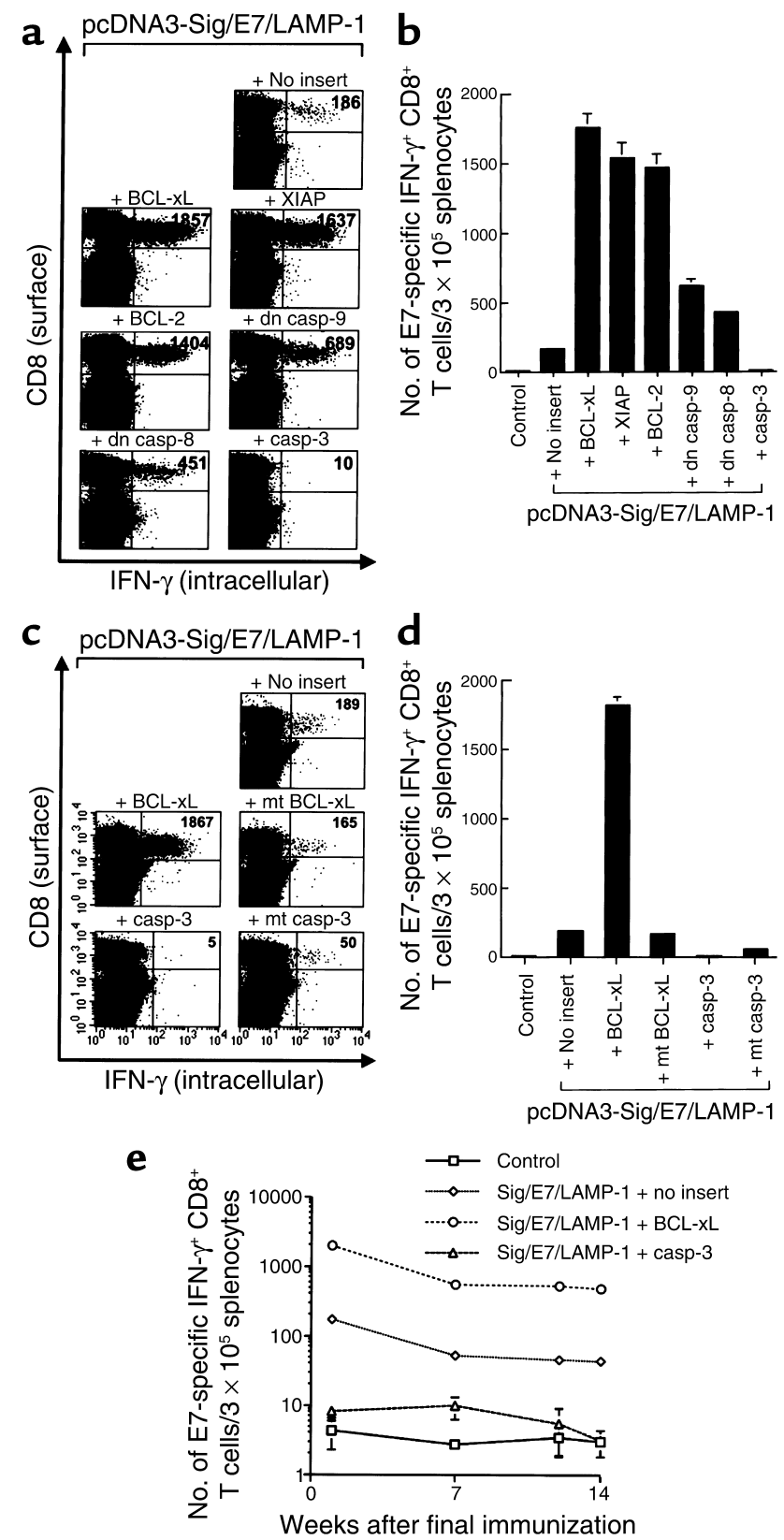

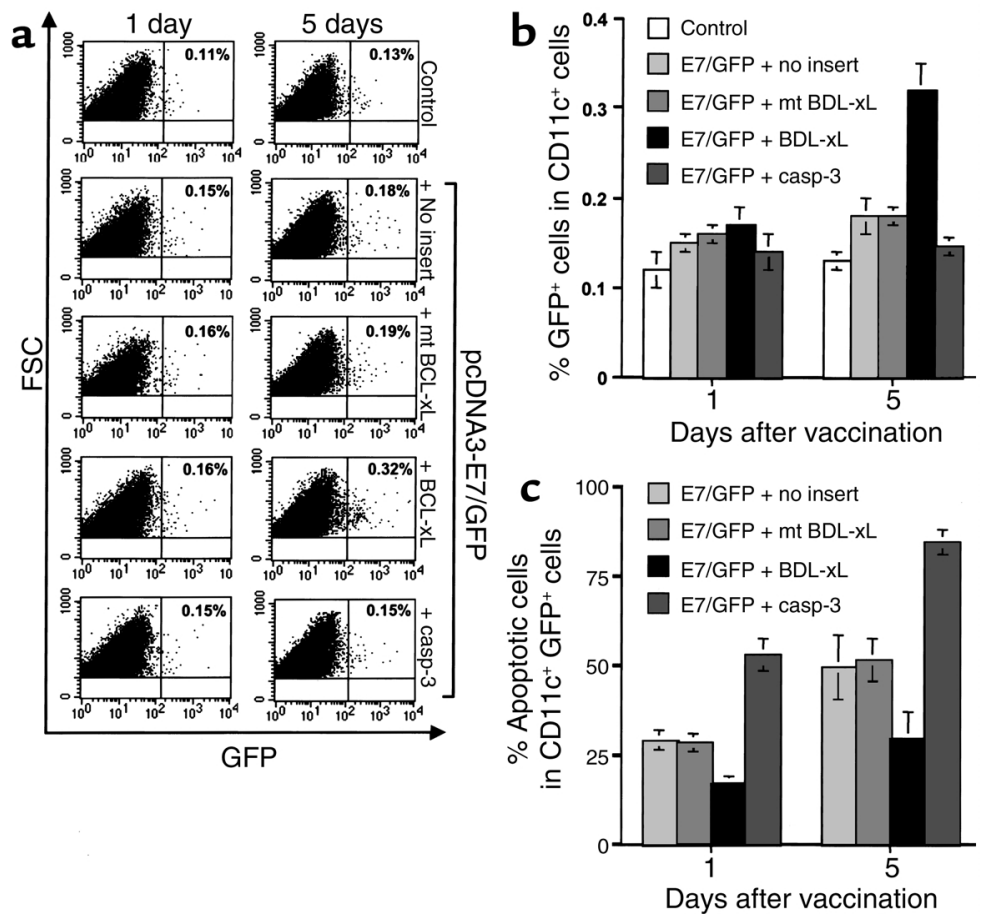

\section{Figure 4}

Characterization of DNA-transfected DCs in the inguinal lymph nodes of vaccinated mice. Mice (three per group) were immunized with pcDNA3-E7/GFP DNA mixed with pSG5-BCL-xL, pSG5-mt BCL-xL, pSG5-caspase-3, or pSG5. The PcDNA3 mixed with pSG5-BCL-xL was used as a negative control. The inguinal lymph nodes were harvested from mice 1 and 5 days after gene gun vaccination. DCs were enriched from a single-cell suspension of lymph nodes using CD11c microbeads. Enriched CD $11 \mathrm{c}^{+}$cells were analyzed in terms of forward scatter versus side scatter; the gated area represents the monocyte population. (a) Representative flow-cytometry data of the percentage of E7/GFP-transfected CD11 $c^{+}$cells among the gated monocytes. The data presented in this figure are from one representative experiment of three performed. (b) Bar graph depicting the percentage of $\mathrm{CD} 11 \mathrm{c}^{+} \mathrm{GFP}^{+}$monocytes among the gated monocytes (mean \pm SD). (c) Bar graph depicting the percentage of apoptotic cells in CD $11 \mathrm{c}^{+} \mathrm{GFP}^{+}$cells (mean $\pm \mathrm{SD}$ ). The percentage of apoptotic cells was determined by staining with annexin $\mathrm{V}$ followed by flow-cytometry analysis using the $\mathrm{CD} 11 \mathrm{c}^{+} \mathrm{GFP}^{+}$cells derived from the draining lymph nodes of various vaccinated mice. casp, caspase; FSC, forward scatter; SSC, side scatter.

BCL-xL demonstrated a significantly lower percentage of apoptotic cells when compared with other vaccinated mice $(P<0.0005$, one-way ANOVA). Thus, our results suggest that coadministration of E7/GFP DNA with DNA encoding an antiapoptotic protein may prolong the survival of DNA-transfected DCs.

CD11c-enriched cells from mice vaccinated with E7/GFP $D N A$ coadministered with DNA encoding BCL- $x L$ enhances activation of an E7-specific $C D 8^{+} T$ cell line. We evaluated the ability of CD11c-enriched cells from the inguinal lymph nodes of the various DNA-vaccinated mice to stimulate INF- $\gamma$ secretion from an E7-specific $\mathrm{CD}^{+} \mathrm{T}$ cell line (23). These CD11c-enriched cells were isolated at 1 or 5 days after the last DNA vaccination and were incubated with an E7-specific T cell line. As shown in Figure 5, CD11c-enriched cells isolated from mice vaccinated with E7/GFP DNA mixed with BCL-xL DNA were more effective in activating the $\mathrm{E} 7$-specific $\mathrm{CD}^{+}$ $\mathrm{T}$ cell line to secrete IFN- $\gamma$ compared with other DNA vaccines, particularly at day $5(P<0.0005$, one-way ANOVA). In comparison, the CD11c-enriched cells isolated from mice vaccinated with E7/GFP DNA mixed with DNA encoding caspase- 3 or no insert did not efficiently activate E7-specific $\mathrm{CD}^{+} \mathrm{T}$ cells on day 5 . These results are consistent with the notion that DNA-transfected DCs from mice vaccinated with E7 DNA coadministered with DNA encoding BCL-xL can enhance activation of E7-specific CD8 ${ }^{+} \mathrm{T}$ cells.

\section{Discussion}

Our study demonstrated that coadministration of antigen-containing DNA with DNA encoding antiapoptotic proteins enhances antigen-specific $\mathrm{CD}^{+} \mathrm{T}$ cell-mediated immune responses and increases the sur- vival of DCs in the draining lymph nodes. In contrast, previous studies have demonstrated that DNA vaccines encoding antigen coexpressed with proapoptotic agents such as Fas (25), or mutant caspases with altered active sites (24), or suicide DNA encoding antigen (26) are able to enhance antigen-specific $\mathrm{T}$ cell immune responses. This apparent inconsistency may be explained by considering the various factors that are involved in these studies, such as the model antigen that is used, the expression vector used, the dose of vaccination, the regimen of vaccination, and the route of administration. Among these factors, the route of administration likely plays an important role in the effects mediated by DNA vaccines encoding apoptotic or antiapoptotic proteins. All of the previous studies using proapoptotic DNA vaccines to enhance vaccine potency employ intramuscular immunization (24-26). In contrast, our study uses intradermal immunization of DNA encoding antiapoptotic factors. Intramuscular immunization most likely targets antigen to myocytes, which are not ideal professional APCs since they do not express costimulatory molecules that are important for efficient activation of $T$ cells. In this setting, the transfection of cells with DNA encoding proapoptotic factors may lead to apoptosis or necrosis, resulting in uptake of antigen by APCs through an exogenous cross-priming pathway, a process involving presentation of exogenous antigen through the MHC class I pathway to $\mathrm{CD} 8^{+} \mathrm{T}$ cells (for review, see refs. 27,28 ). In contrast, intradermal immunization can directly target antigen to Langerhans cells and can facilitate direct presentation of antigen to $T$ cells by DNA-transfected DCs $(1,2)$. It has also been shown that direct presentation plays an key role in antigen presentation to $\mathrm{CD} 8^{+}$ 
$T$ cells after intradermal immunization with a gene gun (2). Our data are consistent with this notion. Our results suggest that inhibition of apoptosis can lead to enhanced survival of DNA-transfected DCs and can result in a significant increase in the number of activated antigen-specific $\mathrm{T}$ cells. These interpretations suggest that various factors involved in vaccination, such as the route of administration, may have a profound effect on the effectiveness of DNA vaccines employing proapoptotic or antiapoptotic factors.

We found that coadministration of antigen-containing DNA with DNA encoding antiapoptotic agents such as BCL-xL resulted in an increased number of antigen-expressing DCs in the draining lymph nodes. In contrast, coadministration with DNA encoding mutant BCL-xL (which has minimal mutations in a region critical to antiapoptotic function) failed to lead to an increase in number of antigen-expressing DCs in the draining lymph nodes (Figure 4b). Furthermore, we observed that mice vaccinated with DNA encoding E7/GFP mixed with DNA encoding BCL-xL demonstrated a significantly lower percentage of apoptotic cells in $\mathrm{CD}_{1} 1 \mathrm{c}^{+} \mathrm{GFP}^{+}$cells derived from the draining lymph nodes when compared with mice vaccinated with DNA encoding E7/GFP mixed with DNA encoding $m t$ BCL-xL (Figure 4c). Taken together, these data strongly suggest that the increase of DCs expressing a specific antigen in the draining lymph nodes is likely due to inhibition of DC apoptosis. A previous study by Nopora et al. also demonstrated that the DCs derived from BCL-2 transgenic mice have a higher survival rate than the DCs from naive mice (29). We could not rule out, however, the possibility that vaccination with DNA encoding antiapoptotic agents may affect DC migration through chemokines or other factors that influence DC homing to the draining lymph nodes after acquisition of antigen in the periphery.

Our results suggest that the increase in the number of antigenexpressing DCs in the lymph nodes may contribute to the enhancement of E7-specific $T$ cell activation. There may be other explanations for enhanced $\mathrm{T}$ cell activation, however, such as qualitative changes in antigen-expressing DCs as a result of vaccination with DNA encoding antiapoptotic factors. For example, bacterial DNA can contain immunostimulatory elements such as cytosine linked to a guanine by a phosphate bond (CpG) islands
(30), which have been shown to cause simultaneous maturation and activation of murine DCs (31) and alter the expression of cytokines such as IL-12 and IFN- $\gamma$ $(30,32)$. CpG islands probably did not play a major role, however, since vaccination with mutant BCL-xL, which had minimal mutations that abrogated antiapoptotic function but relatively unaltered $\mathrm{CPG}$ islands compared with wild-type BCL-xL, failed to enhance E7-specific CD8 ${ }^{+} \mathrm{T}$ cell immune responses. Thus, antiapoptotic function likely plays an important role in the observed increase in the number of antigenexpressing DCs in the draining lymph nodes and in the enhancement of E7-specific $\mathrm{CD}^{+} \mathrm{T}$ cell activation in vaccinated mice.

In this study, we observed that coadministration of DNA encoding BCL-xL with DNA encoding antigen generated the most potent enhancement of antigenspecific $\mathrm{CD}^{+} \mathrm{T}$ cell immune responses among the antiapoptotic proteins that we tested. BCL-xL is considered one of the most potent antiapoptotic proteins. BCL-xL, like BCL-2, has been shown to localize to outer mitochondrial membranes and prevent release of proapoptotic factors from mitochondria such as cytochrome $c$ (33) and Smac/DIABLO (34-36) by a mechanism that is not yet well understood. In addition, BCL-xL may inhibit apoptosis downstream of caspase-8 (37). Thus,
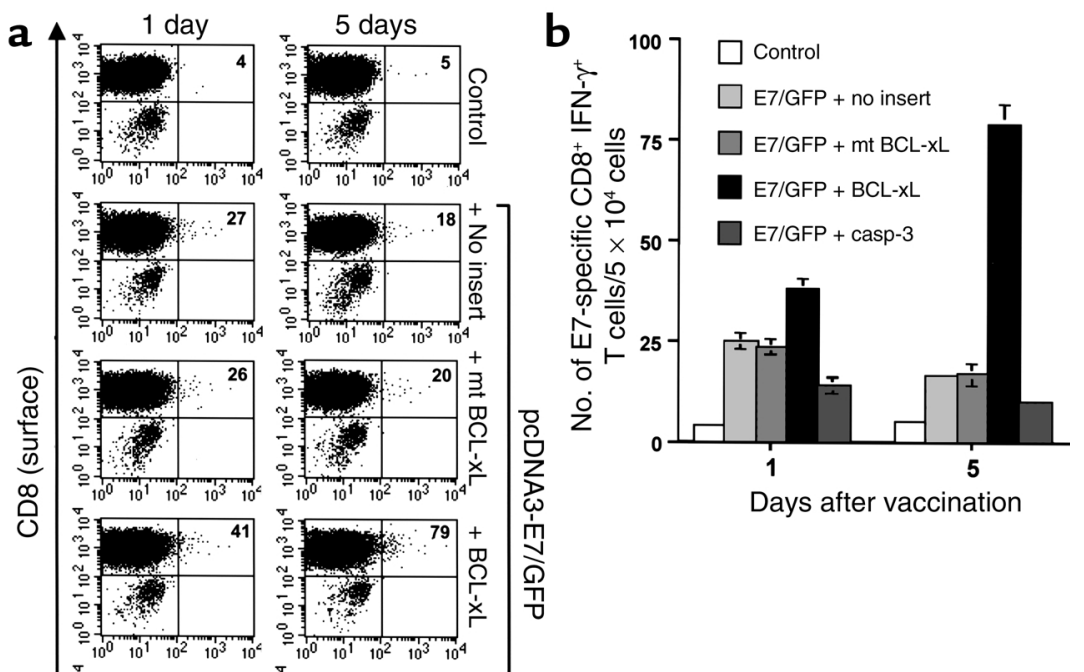

Days after vaccination

\section{Figure 5}

Activation of E7-specific CD8 ${ }^{+} \mathrm{T}$ cells by CD11c-enriched cells isolated from the inguinal lymph nodes of vaccinated mice. Mice (three per group) were immunized and CD11 $c^{+}$cells were enriched as described in the legend for Figure 4. CD11c-enriched cells were incubated with an E7-specific CD8 ${ }^{+} T$ cell line. Cells were then stained for both CD8 and intracellular IFN- $\gamma$. Intracellular cytokine staining and flow-cytometry analysis were performed to determine the number of E7-specific IFN- $\gamma$-secreting CD8 ${ }^{+}$T cells per $5 \times 10^{4}$ cells. (a) Representative flow-cytometry data. The data shown here are from one representative experiment of three performed. (b) Bar graph depicting the number of E7-specific IFN- $\gamma$-secreting CD8 ${ }^{+}$ T cells per $5 \times 10^{4}$ cells (mean \pm SD). 
BCL-xL may inhibit apoptosis at multiple points along the programmed cell death pathways, which explains why it is one of the most potent antiapoptotic factors. In summary, the results of this study demonstrate the usefulness of vaccination with DNA encoding antiapoptotic protein in combination with DNA encoding antigen as an approach to enhance antigen-specific $\mathrm{CD}^{+} \mathrm{T}$ cell immune responses and antigen-specific antitumor effects. Furthermore, the antiapoptosis strategy can also be synergistically applied in combination with other DNA vaccines that encode E7 linked to immunogenic molecules. The promising results of this study encourage the application of this strategy to target other cancers or infectious diseases with known antigens.

\section{Acknowledgments}

We thank Robert J. Kurman and Drew M. Pardoll for helpful discussions. We also thank Ralph Hruban, KenYu Lin, and Richard Roden for critical review of the manuscript. This work was supported by the National Cancer Institute, the Cancer Research Institutes, and the American Cancer Society.

1. Condon, C., Watkins, S.C., Celluzzi, C.M., Thompson, K., and Falo, L.D., Jr. 1996. DNA-based immunization by in vivo transfection of dendritic cells. Nat. Med. 2:1122-1128.

2. Porgador, A., et al. 1998. Predominant role for directly transfected dendritic cells in antigen presentation to CD8+ T cells after gene gun immunization. J. Exp. Med. 188:1075-1082.

3. Ji, H., et al. 1999. Targeting human papillomavirus type $16 \mathrm{E} 7$ to the endosomal/lysosomal compartment enhances the antitumor immunity of DNA vaccines against murine human papillomavirus type 16 E7expressing tumors. Hum. Gene Ther. 10:2727-2740.

4. Chen, C.H., et al. 2000. Enhancement of DNA vaccine potency by linkage of antigen gene to an HSP70 gene. Cancer Res. 60:1035-1042.

5. Hung, C.F., et al. 2001. Cancer immunotherapy using a DNA vaccine encoding the translocation domain of a bacterial toxin linked to a tumor antigen. Cancer Res. 61:3698-3703.

6. Cheng, W.F., et al. 2001. Tumor-specific immunity and antiangiogenesis generated by a DNA vaccine encoding calreticulin linked to a tumor antigen. J. Clin. Invest. 108:669-678.

7. Hung, C.F., et al. 2001. Improving vaccine potency through intercellular spreading and enhanced MHC class I presentation of antigen. J. Immunol. 166:5733-5740.

8. Hung, C.F., et al. 2002. Improving DNA vaccine potency by linking Marek's disease virus type 1 VP22 to an antigen. J. Virol. 76:2676-2682.

9. Boise, L.H., et al. 1993. Bcl-x, a bcl-2-related gene that functions as a dominant regulator of apoptotic cell death. Cell. 74:597-608.

10. Cheng, E.H., Levine, B., Boise, L.H., Thompson, C.B., and Hardwick, J.M. 1996. Bax-independent inhibition of apoptosis by Bcl-XL. Nature. 379:554-556.

11. Vaux, D.L., Cory, S., and Adams, J.M. 1988. Bcl-2 gene promotes haemopoietic cell survival and cooperates with c-myc to immortalize preB cells. Nature. 335:440-442.

12. Hockenbery, D., Nunez, G., Milliman, C., Schreiber, R.D., and Korsmeyer, S.J. $1990 . \mathrm{Bcl}-2$ is an inner mitochondrial membrane protein that blocks programmed cell death. Nature. 348:334-336.

13. Deveraux, Q.L., Takahashi, R., Salvesen, G.S., and Reed, J.C. 1997.
$\mathrm{X}$-linked IAP is a direct inhibitor of cell-death proteases. Nature. 388:300-304.

14. Pan, G., O'Rourke, K., and Dixit, V.M. 1998. Caspase-9, Bcl-XL, and Apaf-1 form a ternary complex. J. Biol. Chem. 273:5841-5845.

15. Muzio, M., et al. 1996. FLICE, a novel FADD-homologous ICE/CED-3like protease, is recruited to the CD95 (Fas/APO-1) death-inducing signaling complex. Cell. 85:817-827.

16. Fernandes-Alnemri, T., Litwack, G., and Alnemri, E.S. 1994. CPP32, a novel human apoptotic protein with homology to Caenorhabditis elegans cell death protein Ced-3 and mammalian interleukin-1 beta-converting enzyme. J. Biol. Chem. 269:30761-30764.

17. Clem, R.J., et al. 2001. c-IAP1 is cleaved by caspases to produce a proapoptotic C-terminal fragment. J. Biol. Chem. 276:7602-7608.

18. Cheng, E.H., et al. 1997. Conversion of Bcl-2 to a Bax-like death effector by caspases. Science. 278:1966-1968.

19. Stennicke, H.R., et al. 1999. Caspase-9 can be activated without proteolytic processing. J. Biol. Chem. 274:8359-8362.

20. Colussi, P.A., Harvey, N.L., Shearwin-Whyatt, L.M., and Kumar, S. 1998. Conversion of procaspase- 3 to an autoactivating caspase by fusion to the caspase-2 prodomain. J. Biol. Chem. 273:26566-26570.

21. Lin, K.Y., et al. 1996. Treatment of established tumors with a novel vaccine that enhances major histocompatibility class II presentation of tumor antigen. Cancer Res. 56:21-26.

22. Lappin, M.B., et al. 1999. Analysis of mouse dendritic cell migration in vivo upon subcutaneous and intravenous injection. Immunology. 98:181-188.

23. Wang, T.L., et al. 2000. Intramuscular administration of E7-transfected dendritic cells generates the most potent E7-specific anti-tumor immunity. Gene Ther. 7:726-733.

24. Sasaki, S., Amara, R.R., Oran, A.E., Smith, J.M., and Robinson, H.L. 2001. Apoptosis-mediated enhancement of DNA-raised immune responses by mutant caspases. Nat. Biotechnol. 19:543-547.

25. Chattergoon, M.A., et al. 2000. Targeted antigen delivery to antigen-presenting cells including dendritic cells by engineered Fas-mediated apoptosis. Nat. Biotechnol. 18:974-979.

26. Leitner, W.W., Ying, H., Driver, D.A., Dubensky, T.W., and Restifo, N.P. 2000. Enhancement of tumor-specific immune response with plasmid DNA replicon vectors. Cancer Res. 60:51-55.

27. Srivastava, P.K., Menoret, A., Basu, S., Binder, R.J., and McQuade, K.L. 1998. Heat shock proteins come of age: primitive functions acquire new roles in an adaptive world. Immunity. 8:657-665.

28. Heath, W.R., and Carbone, F.R. 2001. Cross-presentation, dendritic cells, tolerance and immunity. Annu. Rev. Immunol. 19:47-64.

29. Nopora, A., and Brocker, T. 2002. Bcl-2 controls dendritic cell longevity in vivo. J. Immunol. 169:3006-3014.

30. Sato, Y., et al. 1996. Immunostimulatory DNA sequences necessary for effective intradermal gene immunization. Science. 273:352-354.

31. Sparwasser, T., et al. 1998. Bacterial DNA and immunostimulatory CpG oligonucleotides trigger maturation and activation of murine dendritic cells. Eur. J. Immunol. 28:2045-2054.

32. Hemmi, H., et al. 2000. A Toll-like receptor recognizes bacterial DNA. Nature. 408:740-745.

33. Kharbanda, S., et al. 1997. Role for Bcl-xL as an inhibitor of cytosolic cytochrome c accumulation in DNA damage-induced apoptosis. Proc. Natl. Acad. Sci. U. S. A. 94:6939-6942.

34. Du, C., Fang, M., Li, Y., Li, L., and Wang, X. 2000. Smac, a mitochondrial protein that promotes cytochrome c-dependent caspase activation by eliminating IAP inhibition. Cell. 102:33-42.

35. Verhagen, A.M., et al. 2000. Identification of DIABLO, a mammalian protein that promotes apoptosis by binding to and antagonizing IAP proteins. Cell. 102:43-53.

36. Sun, X.M., Bratton, S.B., Butterworth, M., MacFarlane, M., and Cohen, G.M. 2002. Bcl-2 and Bcl-xL inhibit CD95-mediated apoptosis by preventing mitochondrial release of Smac/DIABLO and subsequent inactivation of XIAP. J. Biol. Chem. 277:11345-11351.

37. Medema, J.P., Scaffidi, C., Krammer, P.H., and Peter, M.E. 1998. Bcl-xL acts downstream of caspase- 8 activation by the CD95 death-inducing signaling complex. J. Biol. Chem. 273:3388-3393. 